\title{
The tolerability of linezolid in the treatment of nontuberculous mycobacterial disease
}

\begin{abstract}
To the Editor:
Nontuberculous mycobacteria (NTM) disease is increasingly common and more prevalent than tuberculosis (TB) in many areas of the world $[1,2]$. Treatment is challenging due to the multidrug-resistant nature of these organisms, and the need for prolonged multidrug therapy [2-4].

Linezolid (Zyvox; Pfizer Inc., New York, NY, USA) is approved for the treatment of drug-resistant, gram-positive bacterial infections, and recent studies suggest that linezolid may be useful in the treatment of multidrug-resistant TB [2-4]. However, linezolid's association with treatment-limiting adverse events, such as cytopenias and neuropathy, complicates its long-term use [3, 5-10].

To our knowledge, no studies have yet evaluated the tolerability of linezolid during therapy for NTM disease $[3,4,11]$.

We conducted a retrospective cohort study at six NTM treatment centres in North America and sought to evaluate tolerability of linezolid in NTM patients who were using linezolid prior to January 2013. All of the included patients either met the American Thoracic Society (ATS)/Infectious Diseases Society of America (IDSA) criteria for pulmonary NTM disease or had extrapulmonary disease (defined as positive NTM soft tissue or sterile site culture). Statistical analyses were performed using Stata version 11.0 (Stata, College Station, TX, USA).
\end{abstract}

We identified 102 NTM patients who met the criteria ( $78 \%$ had pulmonary disease). Most were female $(73 \%)$ and white $(80 \%)$, with a median age of 58 years (range 3-88 years). Common comorbidities included bronchiectasis (54\%), autoimmune disease (23\%) and chronic obstructive pulmonary disease (16\%). Of the 66 pulmonary patients with radiologic data available, 18 (27\%) had cavitary disease and $37(56 \%)$ had nodular disease. Common pathogens included Mycobacterium abscessus (44\%), Mycobacterium avium complex (MAC) (33\%) and Mycobacterium chelonae (14\%). Macrolides ( $\mathrm{n}=81,80 \%)$, aminoglycosides $(\mathrm{n}=45,45 \%)$ and fluoroquinolones $(\mathrm{n}=33,33 \%)$ were commonly used concomitantly and $67(66 \%)$ patients received daily vitamin B6 (75\% received $50 \mathrm{mg}, 12 \%$ received $<50 \mathrm{mg}$ and $11 \%$ received $100 \mathrm{mg}$ ).

Median linezolid therapy duration after initial drug start was 21.4 weeks (range 1-201 weeks) (79\% took $600 \mathrm{mg}$ once daily, $12 \%$ took $300 \mathrm{mg}$ once daily and $5 \%$ took $600 \mathrm{mg}$ twice daily). After therapy had commenced, 46 (45\%) developed adverse events that a clinician attributed to linezolid after a median 19.9 weeks (range $0.1-107$ weeks). Peripheral neuropathy developed in $24(24 \%)$ patients in a median of 38 weeks (range 2-233 weeks). Other adverse events attributed to linezolid included gastro-intestinal intolerance $(n=9,9 \%)$, anaemia $(n=8,8 \%)$ and thrombocytopenia $(n=6,6 \%)$. Among those who used vitamin B6 concomitantly, cytopenias and peripheral neuropathy occurred in $8(12 \%)$ and $16(24 \%)$ individuals, respectively, compared with $4(11 \%)$ and $8(23 \%)$ individuals among those not taking vitamin B6. Furthermore, the proportion of patients developing linezolid-attributable adverse events was similar between pulmonary and extrapulmonary disease ( $47 \%$ versus $41 \%)$, between those using and not using rifampin (33\% versus $48 \%$ ), and between those with MAC and those with M. abscessus (37\% versus $51 \%$ ).

Of the patients with linezolid-attributable adverse events, most (87\%) stopped therapy, with treatment discontinuation occurring at a median of 20 weeks (range 1-109 weeks, incidence rate 57.1 per 100 person-years) (fig. 1). Of these 40 patients, 17 (42.5\%) later resumed linezolid at either $300 \mathrm{mg}$ once daily $(\mathrm{n}=6), 600 \mathrm{mg}$ once daily $(\mathrm{n}=9), 600 \mathrm{mg}$ twice daily $(\mathrm{n}=1)$ or an unknown dosage $(\mathrm{n}=1)$ for a median duration of 17.3 weeks (range $0.4-95.3$ weeks).

The proportion of patients who developed linezolid-attributable adverse reactions in this study is similar to that reported during long-term use of linezolid as part of multidrug therapy for multidrug-resistant TB. A case series by SCHECTER et al. [4] found that the use of $600 \mathrm{mg}$ once daily of linezolid led to lower rates of treatment discontinuation than twice daily dosing, while vitamin B6 was given to mitigate haematological 


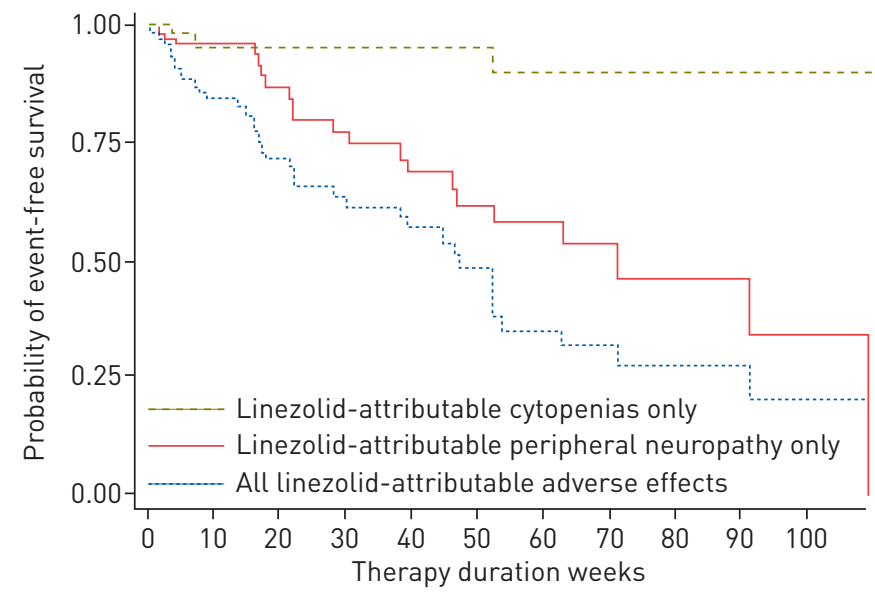

At risk $n$
FIGURE 1 Kaplan-Meier curve demonstrating the proportion of patients who discontinued linezolid therapy due to an adverse event attributed to linezolid.

adverse events. In our study, approximately two-thirds of patients received vitamin B6 concomitantly. We used the Chi-squared test to examine the correlation between various patient factors, including the use of vitamin B6 and the occurrence of linezolid-attributable adverse events reported by the treating clinician; we found no difference in the proportion of patients developing cytopenias ( $12 \%$ in users versus $11 \%$ in non-users; $\mathrm{p}=0.9$ ) or peripheral neuropathy ( $24 \%$ in users versus $23 \%$ in non-users $\mathrm{p}=0.9$ ) between vitamin B6 users and non-users. Balancing the risk-benefit ratio of long-term linezolid use requires identification of a dose that effectively achieves clinical benefit with acceptably low toxicity. In our study, most patients used $600 \mathrm{mg}$ once daily dose, making comparison with a lower or higher dose impossible. For drug-resistant TB, some studies suggest that adverse events are more common with a 600-mg daily dosage than lower doses [3]. Similarly, in a systematic review of literature on linezolid-containing regimens used to treat resistant TB performed by Cox and FORD [8], a trend towards fewer adverse events was described with a linezolid dose of $\leqslant 600 \mathrm{mg}$ daily than higher doses. However, minimum inhibitory concentrations for linezolid with TB are generally lower than for NTM, making it unclear whether once daily dosing or doses $<600 \mathrm{mg}$ would provide clinical benefit [12-14]. Further randomised control trials should evaluate the tolerability and efficacy of $600 \mathrm{mg}$ once daily linezolid in NTM disease.

The retrospective and nonrandomised nature of our study restricted our ability to draw firm conclusions regarding the tolerability of linezolid in the context of multidrug NTM therapy. In addition, adverse events attributed to linezolid by the treating physician may in fact have been due to other therapies or patient conditions that existed concurrently. Lastly, we were limited in making safety comparisons between different linezolid dosages as the majority of patients in our study used $600 \mathrm{mg}$ once daily dosing.

In summary, we have documented that linezolid can be used for long durations in the multidrug therapy of NTM disease. However, adverse events necessitating drug discontinuation are not uncommon, occurring in $>40 \%$ of patients regardless of concomitant vitamin B6 use. To our knowledge, this is the largest case series published to date to describe the use and tolerability of linezolid in a large number of patients with pulmonary NTM disease. Larger studies are necessary to ascertain the efficacy and long-term tolerability of linezolid, as well as the potential protective effects of concomitant vitamin B6.

@ERSpublications

Linezolid can be tolerated long-term in the treatment of nontuberculous mycobacterial disease http://ow.ly/FilUm

Kevin L. Winthrop ${ }^{1}$, Jennifer H. Ku ${ }^{1}$, Theodore K. Marras ${ }^{2}$, David E. Griffith ${ }^{3}$, Charles L. Daley ${ }^{4}$, Kenneth N. Olivier ${ }^{5}$, Timothy R. Aksamit ${ }^{6}$, Cara D. Varley ${ }^{1}$, Katherine Mackey ${ }^{1}$ and D. Rebecca Prevots ${ }^{5}$

${ }^{1}$ Divisions of Infectious Diseases, Public Health and Preventive Medicine, Oregon Health and Science University, Portland, OR, USA. ${ }^{2}$ University of Toronto, Toronto, Canada. ${ }^{3}$ University of Texas at Tyler, Tyler, TX, USA. ${ }^{4}$ National Jewish Health, Denver, CO, USA. ${ }^{5}$ National Institute Allergy and Infectious Diseases, Bethesda, MD, USA. ${ }^{6}$ Mayo Clinic, Rochester, MN, USA.

Correspondence: Kevin L. Winthrop, Divisions of Infectious Diseases, Public Health and Preventive Medicine, Oregon Health and Science University, Mailcode CEI, 3375 Terwilliger Blvd, Portland, OR 97239, USA.

E-mail: Winthrop@ohsu.edu 
Received: July 012014 | Accepted after revision: Nov 252014 | First published online: Jan 222015

Statement of interest: Disclosures can be found alongside the online version of this article at erj.ersjournals.com

Support statement: This study was supported by Investigator Initiated Research funding from Pfizer Inc. and in part by the Intramural Research Programme (National Institute of Allergy and Infectious Diseases (NIAID)). Funding information for this article has been deposited with FundRef.

\section{References}

1 Adjemian J, Olivier KN, Seitz AE, et al. Prevalence of nontuberculous mycobacterial lung disease in US medicare beneficiaries. Am J Respir Crit Care Med 2012; 185: 881-886.

2 CDC. Reported tuberculosis in the United States, 2012. Atlanta, US Department of Health and Human Services, 2013.

3 Lee M, Lee J, Carroll MW, et al. Linezolid for treatment of chronic extensively drug-resistant tuberculosis. $N$ Engl J Med 2012; 367: 1508-1518.

4 Schecter GF, Scott C, True L, et al. Linezolid in the treatment of multidrug-resistant tuberculosis. Clin Infect Dis 2010; 50: 49-55.

5 Migliori GB, Eker B, Richardson MD, et al. A retrospective TBNET assessment of linezolid safety, tolerability and efficacy in multidrug-resistant tuberculosis. Eur Respir J 2009; 34: 387-393.

6 Sotgiu G, Centis R, D'Ambrosio L, et al. Efficacy, safety and tolerability of linezolid containing regimens in treating MDR-TB and XDR-TB: systematic review and meta-analysis. Eur Respir J 2012; 40: 1430-1442.

7 Sotgiu G, Centis R, D’Ambrosio L, et al. Linezolid to treat extensively drug-resistant TB: retrospective data are confirmed by experimental evidence. Eur Respir J 2013; 42: 288-290.

8 Cox H, Ford N. Linezolid for the treatment of complicated drug-resistant tuberculosis: a systematic review and meta-analysis. Int J Tuberc Lung Dis 2012; 16: 447-454.

9 Vinh DC, Rubinstein E. Linezolid: a review of safety and tolerability. J Infect 2009; 59: Suppl. 1, S59-S74.

10 Bishop E, Melvani S, Howden BP, et al. Good clinical outcomes but high rates of adverse reactions during linezolid therapy for serious infections: a proposed protocol for monitoring therapy in complex patients. Antimicrob Agents Chemother 2006; 50: 1599-1602.

11 Koh WJ, Kang YR, Jeon K, et al. Daily $300 \mathrm{mg}$ dose of linezolid for multidrug-resistant and extensively drug-resistant tuberculosis: updated analysis of 51 patients. J Antimicrob Chemother 2012; 67: 1503-1507.

12 Rey-Jurado E, Tudo G, de la Bellacasa JP, et al. In vitro effect of three-drug combinations of antituberculous agents against multidrug-resistant mycobacterium tuberculosis isolates. Int J Antimicrob Agents 2013; 41: 278-280.

13 Guna R, Munoz C, Dominguez V, et al. In vitro activity of linezolid, clarithromycin and moxifloxacin against clinical isolates of mycobacterium kansasii. J Antimicrob Chemother 2005; 55: 950-953.

14 Wallace RJJr, Brown-Elliott BA, Ward SC, et al. Activities of linezolid against rapidly growing mycobacteria. Antimicrob Agents Chemother 2001; 45: 764-767.

\section{Serious adverse events during a 6-min walk test in patients with pulmonary hypertension}

\section{To the Editor:}

The 6-min walk test (6MWT) is well standardised and, as per current guidelines, is deemed safe to perform in a range of patients [1]. In pulmonary arterial hypertension (PAH), 6-min walk distance (6MWD) is an important predictor of mortality and morbidity [2]. Moreover, changes in the 6MWD have been the primary end-point of most pivotal randomised controlled trials that have assessed the clinical response to specific pharmacological therapies in PAH [2].

To date there have been no published reports about the serious adverse events associated with the 6MWT, e.g. death, life-threatening events, inpatient hospitalisation or prolongation of existing hospitalisation, persistent or significant incapacity, and substantial disruption to the ability to conduct normal life [3]. Perhaps the best reported study that supports the safety of the 6MWT is that of ENRIGHT et al. [4], which was performed in over 2000 elderly subjects ( $77 \pm 4$ years of age) with a range of comorbidities. The authors reported no serious adverse events during the 6MWT, and whilst some participants stopped the test with chest pain, no emergency evaluation or therapy was required. More recently, in a review of 741 stable lung disease patients, 